\title{
GESTIÓN DE RECURSOS HUMANOS EN LA SATISFACCIÓN DE LOS USUARIOS EN CONSULTA EXTERNA DE LA UBAP MANTARO
}

Autores:

Tapia Huamán, R.

\section{RESUMEN}

\section{RESUMEN}

El trabajo de investigación Gestión de recursos humanos en la satisfacción de los usuarios en consulta externa de la UBAP Mantaro tiene como objetivo determinar la influencia de la gestión de recursos humanos en la satisfacción del usuario para lo cual se seleccionaron 274 pacientes de consulta externa y mediante una metodología descriptiva explicativa donde se determinó que la gestión de recursos humanos influye positivamente en la satisfacción del usuario, el tiempo de espera influye directamente en la satisfacción del usuario y el nivel de instrucción de los asegurados influye directamente en la percepción de la satisfacción del usuario en la consulta externa de la UBAP Mantaro, por lo tanto se debe destinar y gestionar los recursos humanos capacitados en los procesos de selección, diseñar un programa de mejoramiento continuo de la calidad para optimizar procesos para disminuir el tiempo de espera , mejorar los canales de comunicación del personal de salud según el nivel de conocimiento de los usuarios y se debe incluir procesos de medición de satisfacción del usuario para conocer la percepción de la atención en los servicios recibidos.

Palabras Claves: Atención, satisfacción del usuario, mejora de procesos, gestión de recursos humanos en salud

\section{SUMMARY}

The research Human resources management in the user satisfaction of outpatient Mantaro UBAP aims to determine the influence of human resource management in customer satisfaction for which we selected 274 outpatients and by explanatory descriptive methodology which determined that the management of human resources positively influences customer satisfaction, waiting time directly affects user satisfaction and educational attainment of the insured directly influences the perception of user satisfaction in the outpatient clinic of the Mantaro UBAP therefore must allocate and manage human resources in the selection process, design a program of continuous quality improvement to optimize processes to reduce waiting times, improve channels of communication of health 
workers by level of knowledge of users and processes should include measurement of user satisfaction to know the perception of care in the s e r vices received. Keywords: Attention, user satisfaction, process improvement, human resource management in health

\section{INTRODUCCIÓN}

La Gestión de recursos humanos es la variable que incide directamente en el grado de satisfacción del usuario externo, por ello es necesario tener en cuenta las características generales del personal que labora en la UBAP Mantaro, para poder dimensionar su participación directa en la atención.

La satisfacción del paciente se considera uno de los valores deseables en la prestación de un servicio, lo cual se convierte en un objetivo estratégico de la Institución prestadora de servicios de salud.

La satisfacción de los pacientes está asociada con las características de la prestación de los servicios de salud. A pesar de las numerosas investigaciones que existe con relación a la satisfacción del paciente con la atención de salud. La satisfacción es un concepto que no está suficientemente definido a nivel teórico, y ha sido difícil desarrollar un modelo conceptual comprensivo. Sin embargo, el concepto incluye básicamente una evaluación individual de la experiencia en el cuidado de la salud comparada con un concepto individual estándar.
Para la profesión es importante promover estándares altos de desempeño entre los profesionales de salud. La autorregulación profesional basada en la responsabilidad pública que implica prestar un servicio de salud, podría ser la mejor manera de asegurar la protección de los pacientes de la negligencia en salud. El estado y los pacientes tienen una participación activa en la vigilancia de la calidad del servicio.

\section{CAPITULO I}

\section{PLANTEAMIENTO DEL PROBLEMA}

\section{OBJETIVOS}

\subsection{Objetivo general}

a. Determinar la influencia de la gestión de recursos humanos en la satisfacción del usuario en consulta externa de la UBAP Mantaro de la Red Asistencial Junín Essalud, con la finalidad de plantear políticas de mejora continua

\subsection{Objetivos específicos:}

a. Especificar la influencia del tiempo de espera en la satisfacción del usuario en consulta externa de la UBAP Mantaro de la Red asistencial Junín Essalud.

b. Señalar la influencia del nivel de instrucción de los asegurados en la calidad de atención en los servicios de consulta externa de la UBAP Mantaro de la Red Asistencial Junín Essalud.

\section{CAPITULO II}

\subsection{METOdOLOGÍA}




\subsubsection{Descriptivo y explicativo}

Por cuanto se especifica todo el marco teórico de la gestión de los recursos humanos para que el servicio de consulta externa de la Unidad básica de atención primaria Mantaro Essalud preste servicios de calidad y se busca especificar las propiedades importantes de personas atendidas mediante un análisis.

\subsection{TIPODE INVESTIGACIÓN}

Por el tipo de investigación, el presente estudio reúne las condiciones metodológicas de una investigación aplicada en razón que se utilizaron conocimientos de la ciencia administrativas a fin de aplicarlas en el proceso de Gestión de Recursos humanos en los servicios de salud.

\subsection{NIVEL DE INVESTIGACIÓN}

De acuerdo a la naturaleza el estudio de investigación, reúne por el nivel de las características un estudio Descriptiva explicativo.

\subsection{LUGAR Y PERIODO DE LA INVESTIGACIÓN}

El trabajo de investigación se llevó a cabo en la Unidad Básica de Atención Primaria Mantaro de la Red Asistencial Junín Essalud en los meses de octubre a diciembre 2011.

\subsection{POBLACIÓN OUNIVERSO}

Pacientes adultos que acuden al servicio de consulta externa (población de 800) de la UBAP
Mantaro de la red asistencial Junín.

2.6 MUESTRA (Tipo de Muestreo, y

Tamaño de la Muestra)

La población está conformada por 800 pacientes que ingresan al servicio de consulta externa de la UBAP Mantaro ESSALUD durante los meses de octubre a diciembre del 2011. La muestra estará constituida por 274 pacientes, tamaño de muestra resulta de la siguiente operación estadística:

\subsection{TÉCNICAS DE RECOLECCIÓN,} VALIDEZ Y CONFIABILIDAD DEL INSTRUMENTO UTILIZADO

Los datos obtenidos con la técnica de la encuesta serán tabulados, analizados e interpretados con ayuda del software estadístico SPSS, (Paquete Estadístico para

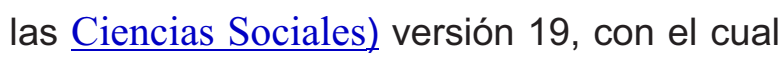
se elaborarán y demostrarán resultados con medidas de tendencia central y variabilidad, así como la prueba de hipótesis con el coeficiente de correlación de Pearson.

Para la validación de la hipótesis se utilizará la $\mathrm{CHI}$ cuadrado por las características cualitativas de las variables.

\section{CAPITULO III}

\subsection{RESULTADOS Y CONSTRUCCION DE} HIPOTESIS

La hipótesis general del trabajo está formulada de la siguiente manera: 
"La Gestión de Recursos humanos influye positivamente en la satisfacción del usuario en consulta externa de la UBAP Mantaro de la Red Asistencial Junín Es salud".

Se puede advertir que si existe un resultado favorable en su salud, esto es producto por lo general de un esmero conjunto de la gestión de los recursos humanos de la UBAP Mantaro, por lo que de ser así el grado de satisfacción del usuario externo debe ser mayor. Como se puede notar, el valor de la chi cuadrado es menor a $\mathbf{0 . 0 5}$ lo que nos permite afirmar de manera categórica que si existe una relación significativa entre la gestión de recursos humanos y la satisfacción de los usuarios externos. La hipótesis general está validada porque existe una relación significativa demostrada con la chi cuadrado y que dicha relación es positiva aunque no alta por el coeficiente de correlación.

Respecto a la primera hipótesis específica que afirma lo siguiente:

\section{"El tiempo de espera influye directamente en la} satisfacción del usuario en consulta externa de la UBAP Mantaro de la Red Asistencial Junín Essalud" Se puede apreciar que el grado de significancia es mayor a 0.05 que demuestra que no existe una relación significativa entre el tiempo de espera y la satisfacción del usuario, es decir, al usuario no le interesa esperar si la atención es al menos regular. El coeficiente de correlación es positivo.

Respecto a la segunda hipótesis específica que afirma lo siguiente:

"El nivel de instrucción de los asegurados influye directamente en la percepción de la satisfacción del usuario en la consulta externa de la UBAP Mantaro de la Red Asistencial Junín Essalud". Siendo el valor de la chi cuadrado menor a 0.05 demuestra que existe relación directa entre las variables, por lo que se valida la hipótesis, pero para apreciar la correlación se calculó el coeficiente de Pearson que arrojó el siguiente resultados.

\section{DISCUSION:}

En la investigación realizada, demuestra que la Gestión de recursos humanos influye positivamente en la satisfacción del usuario, el tiempo de espera influye directamente en la satisfacción del usuario, el nivel de instrucción de los asegurados influye directamente en la percepción de la satisfacción del usuario ,así mismo se demuestra que si los ambientes están limpios la percepción de los usuarios, es que sienten mayor satisfacción en su atención en consulta externa y la atención del médico y la enfermera es fundamental en la percepción de la satisfacción del usuario externo en los servicios de salud de la UBAP Mantaro Essalud.

La Gestión de recursos humanos se inicia con un nuevo liderazgo y por ende una nueva filosofía. Este nuevo liderazgo no solo debe manifestarse con un compromiso firme, constante con claridad de visión, amplia comunicación, convicción de cambio para adoptar una misión acertada. Estos resultados se asemejan a los trabajos realizados por: 
Luis Tipacti "Evaluación de la calidad de la atención de los servicios de la consulta externa en los hospitales públicos de Lima Metropolitana y Callao"(2000). Las conclusiones de la investigación demuestran que el $69 \%$ de los pacientes manifestaron que la atención recibida es buena, mientras que el $60 \%$ de los pacientes manifestaron que las instalaciones de los consultorios externos eran buenas y cómodas. Mientras que el $80 \%$ de los pacientes lo que más le molesta al acudir a los consultorios externos es la pérdida de tiempo y las colas. Así el $82 \%$ señala que debe existir un personal que los oriente al ingreso del hospital. En contraparte el $15 \%$ del personal médico opinaron que las condiciones de trabajo eran buenas y que el $37 \%$ estaban satisfechos con el trabajo que realizan.

Dra., Martha Morales Rivas y Dra. Carola Carvajal Garcés Residentes de Medicina Familiar realizaron el estudio "Percepción de la calidad de atención por el usuario de consulta externa del centro de salud villa candelaria (2003).

Se puede observar que de los 27 usuarios que esperaron 60 minutos, $12(44 \%)$ se quejaron de tiempo de espera prolongado. El porcentaje de quejas se fue incrementando a medida que el tiempo de espera fue mayor; así los que esperaron 75 minutos, se quejaron el $71 \%$, los que esperaron 90 minutos, se quejaron el $70 \%$. Paradójicamente los que esperaron por más tiempo, no se observó que su porcentaje de queja aumentara, aunque se mantuvo alto (67\%).De los usuarios que se quejaron de tiempo de espera prolongado, 21 $(33 \%)$ considera que no se les da atención de calidad

Jesús Salvador Fragoso Bernal, Samuel Efrén Orrico Torres, Modesta Flores Vásquez, Constantino Quiroz Pérez, Araceli Amada Sandoval Priego . Instituto Mexicano realizaron el estudio de investigación Tiempo de espera en el primer nivel para la población asegurada por el IMSS (2003). Las variables independientes fueron las características sociodemográficas de los usuarios, características de la atención y expectativas del usuario sobre el tiempo ideal de espera y prioridad de calidad. Se consideraron dos grupos: usuarios que esperaron máximo 30 minutos, y usuarios que esperaron más de ese tiempo.

Osvaldo Bedoya, desarrolló un estudio titulado: "La nueva gestión de personas y su evaluación de desempeño en empresas competitivas", Universidad Nacional Mayor de San Marcos(2003).. La gestión de personas, en esta nueva concepción, las personas dejan de ser simples recursos (humanos) organizacionales para ser abordadas como seres dotados de inteligencia, personalidad, conocimientos, habilidades, destreza, aspiraciones y percepciones singulares. El estudio de la función de los recursos humanos y del proceso de gestión 
de evaluación de desempeño, así como de su adecuación a los nuevos tiempos, constituye un gran desafío que las empresas deberán afrontar decididamente en los escenarios de mercados globalizados, si desean ser competitivos y permanecer en ellos.

\section{CONCLUSIONES}

1. La gestión de recursos humanos influye positivamente en la satisfacción del usuario en consulta externa de la UBAP Mantaro, demostrada con la chi cuadrado que arroja un valor de significancia menor al 0.05 que indica que entre los recursos humanos y la satisfacción del usuario existe relación directa, de manera que una eficiente gestión repercutirá en la mayor satisfacción del usuario.

2. El tiempo de espera es un factor importante en la satisfacción del usuario externo, sin embargo, el trabajo demostró que la relación cualitativa entre tiempo de espera y satisfacción no es significativo para el usuario, ya que existen otros factores más relevantes como la calidad de atención, que implica la valoración de los profesionales de la salud de la UBAP Mantaro de la red asistencial Junín Essalud, por lo tanto el usuario prefiere esperar pero ser atendido por los especialistas de la salud..
3. Se concluye que el nivel de instrucción de los asegurados influye directamente en la percepción de la satisfacción del usuario en la consulta externa de la UBAP Mantaro de la red asistencial Junín Essalud.

4. La atención que brinda el personal de enfermería y medicina a los pacientes de la UBAP Mantaro en consulta externa es fundamental en la percepción de la satisfacción de la atención recibida, debido al grado de especialización que es valorada por el usuario externo

\section{RECOMENDACIONES}

1. Se debe realizar un seguimiento a la gestión de los recursos humanos de manera permanente, para mantener el nivel de satisfacción favorable en el usuario externo de la UBAP Mantaro mediante las encuestas de satisfacción en la atención del servicio y de esta manera monitorizar y evaluar los resultados periódicamente para establecer la mejora continua de los procesos de atención.

2. Desarrollar mecanismos de información más agresivos de orientación al usuario externo para mejorar su atención, haciendo notar el grado de profesionalismo y la especialización del personal, ya que el tiempo de espera no es 
un factor que incide en la satisfacción del usuario externo de la UBAP Mantaro.

3. Mantener de manera sostenida la capacitación y perfeccionamiento del personal de la salud de la UBAP Mantaro, ya que es un factor determinante en la satisfacción del usuario externo, que mantiene una valoración cualitativa de la especialización en la atención, por ello la permanente calificación coadyuvará en una mayor percepción de la calidad de atención.

4. La atención que brinda el personal de enfermería y medicina a los pacientes de la UBAP Mantaro en consulta externa tiene un nivel de significancia lo cual es fundamental en la percepción de la satisfacción de la atención recibida, sin embargo se debe establecer mecanismos de evaluación permanente al personal para mantener el grado de satisfacción del usuario.

\section{REFERENCIA BIBLIOGRAFÍA}

1. Alonso, J. Gestión de la calidad. Editorial Santillana. España. (1996)

2. Alva J. El contrato administrativo de servicios Lima Perú. (2008)

3. Arsenjo M. Gestión diaria del Hospital.
Editorial Norma. México. (2002)

4. Avgrafoff, Boris. Sistemas de gestión de la producción. Procesos de gestión de la producción. Ibérico Europa de ediciones S.A. Madrid. (2005).

5. Beltrán, Eduardo. Reclutamiento y Selección. Editorial Siglo XXI. Argentina. (1998)

6. Bohlander, George, Sherman, Arthur, \& Snell, Scott. Administración de recursos humanos. Cengage Learning Editores. . (2001)7. Brito, Pedro. Desafíos y problemas de la Gestión de Recursos Humanos de Salud en las Reformas Sectoriales. Organización Panamericana de la Salud. Brasil. (2002)

8. Chiavenato, Idalberto Administración de recursos humanos. México. McGraw-Hill. (2005).

9. Centro de Gestión Hospitalaria Bogotá D.C. Colo $\mathrm{mbi}$ a $S u r a m e ́ r i c a$ http://www.cgh.org.co/temas/2010/calidadensalu d.php

10. Covey Stephen. El Liderazgo Centrado en Principios. Editorial Paidós, Mexicana S.A. (2006)

11. Deming, W. E. Calidad, productividad y competitividad. La salida de la crisis. Díaz de Santos. Madrid. (1989)

12. Gutiérrez, R. La satisfacción del paciente como medida de la evaluación de la calidad. Servicio de Salud de Castilla La Mancha. España. (2003) 
13. Huamán, L. Los lineamientos de política nacional para el desarrollo de los recursos humanos de salud. Ministerio de Salud. Dirección General de Políticas, Regulación y Gestión del Desarrollo de los Recursos Humanos. Lima. (2005)

14. Lineamientos operativos del sistema de monitoreo para la gestión de calidad primer nivel . Cruzada nacional por la calidad. (2002).

15. Ministerio de Salud-MINSA Lineamientos de políticas de recursos humanos en salud Lima Perú. (2005).

16. Morales, S. Sistematización del modelo organizacional del Hospital España de Chinandega. CIES. Managua. España. (1998).

17. Padilla, Mónica. Planificación de los recursos humanos y reformas del sector salud. Programa de Desarrollo de Recursos Humanos de la Organización Panamericana de la Salud. Cuba. . (2002)

18. Retamal Moya Gonzalo, Estrategias de Ens e $\tilde{n} a n z a$ $\mathrm{Ch}$ i I e http://www.leonismoargentino.com.ar/2010/INST 229.htm.

19. Seto, D. De la Calidad de Servicio a la Fidelidad del Cliente. Editorial Enciclopedia. Madrid. (2004).

20. Temes, J. Manual de gestión hospitalaria. Editorial Interamericana. España. (1992)
21. Valarie A. Zeithaml; A. Parasuraman y Leonard L. Berry. Calidad en la Gestión de Servicios-como Lograr el Equilibrio. Madrid. Ediciones Díaz de Santos. (1992).

Fecha de recepción: 30/03/2013 Fecha de aprobación: 08/04/2013 Correspondencia: Rosario tapia Huamán 